\title{
Gestión del Tiempo en 12 Salas Chilenas de Kindergarten: Recreo, Colación y Algo de Instrucción
}

\section{Time Management in 12 Chilean Kindergarten Classrooms: Recess, Snack and a Little Teaching}

\author{
Katherine Strasser, María Rosa Lissi y Macarena Silva \\ Pontificia Universidad Católica de Chile
}

\begin{abstract}
Este estudio examinó la gestión del tiempo en 12 salas de kindergarten chilenas de la Región Metropolitana pertenecientes a 9 establecimientos de diversos tipos de dependencia y niveles socioeconómicos (NSE). Para ello se realizó un total de 33 observaciones en las 12 salas (promedio de duración de 197 minutos) y se determinó en qué tipo de actividades se invertía el tiempo. Más de la mitad de la jornada en las salas de la muestra se dedicó a actividades no instruccionales, como juego inestructurado, colación (merienda) y manejo conductual. El tiempo dedicado a actividades instruccionales no se distribuyó de acuerdo a los hallazgos actuales sobre las actividades más productivas para el desarrollo de los niños. Estos resultados fueron independientes del tipo de dependencia y NSE de los establecimientos.

Palabras clave: educación preescolar, kindergarten, lenguaje, enseñanza de lectura, gestión del tiempo en el aula.

This study examined time management in 12 kindergarten classrooms from 9 Chilean schools with diverse sources of funding and socioeconomic status (SES). We conducted 33 observations in the 12 classrooms (average duration 197 minutes), and determined the amount of time devoted to different activities. More than half of the time in these classrooms was spent in non-instructional activities such as recess, snack, and managing the children's behavior. Additionally, the distribution of instructional time is not in accordance with current findings regarding the activities that are more fruitful for children's development. These results are independent of the schools' source of funding and SES.
\end{abstract}

Keywords: preschool education, kindergarten, language, literacy, time management in kindergarten.

Introducción

La educación preescolar ha sido objeto de una creciente atención, tanto por parte de educadores como de psicólogos, debido al impacto que esta ha mostrado tener tanto en el desarrollo cognitivo y socio emocional de los niños como en su futuro éxito escolar. Los estudios internacionales han mostrado que diversas características de la atención que reciben los niños en la etapa preescolar -cantidad de horas, cantidad y calidad del lenguaje dirigido al niño, número de niños por adulto, materiales, formación de las educadoras, entre otras- se relacionan con variables de desarrollo lingüístico, cognitivo y emocional en forma concurrente y también en forma longitudinal (National Institute of Child Health and Human Development [NICHD], 2000). Además de estos hallazgos generales sobre el efecto del cuidado temprano en el desarrollo psicológico, investigadores interesados en el éxito escolar posterior han encontrado que también ciertas características de la educación recibida en los últimos años de la educación preescolar -a partir de los cuatro años, lo que en Chile se conoce como prekinder y kinder- pueden tener un impacto en ciertas habilidades relacionadas con la adquisición de la lectoescritura en los primeros años de la enseñanza primaria (Connor, Morrison \& Slominski, 2006; Strasser \& Lissi, en prensa).

Katherine Strasser, Escuela de Psicología, Pontificia Universidad Católica de Chile, Santiago, Chile.

María Rosa Lissi, Escuela de Psicología, Pontificia Universidad Católica de Chile, Santiago, Chile. Macarena Silva, Escuela de Psicología, Pontificia Universidad Católica de Chile, Santiago, Chile.

La correspondencia relativa a este artículo debe ser dirigida a Katherine Strasser, Escuela de Psicología, Pontificia Universidad Católica de Chile, Avda. Vicuña Mackenna 4860, Macul, Santiago, Chile. E-mail: kstrasse@uc.cl

Este estudio fue posible gracias al financiamiento del Fondo Nacional de Ciencia y Tecnología (FONDECYT), Proyecto ${ }^{\circ}$ 1040757. 
A partir de los estudios internacionales, hoy sabemos que el efecto positivo de la educación preescolar sobre el desarrollo cognitivo y el rendimiento escolar se encuentra estrechamente ligado a la calidad del programa que se imparte (NICHD, 2000). Rolla y Rivadeneira (2006) plantean que existen elementos estructurales y de proceso asociados a la calidad de la educación preescolar impartida. Entre los elementos estructurales se encontraría el número de alumnos por profesor y la existencia de una adecuada fiscalización que asegure que se cumplan ciertas normativas básicas como seguridad, higiene y nutrición. A su vez, es recomendable contar con educadores bien preparados y en condiciones laborales adecuadas, junto con la existencia de normas sobre los materiales, libros y juguetes necesarios (NICHD, 2000). En la otra arista, algunos elementos importantes de proceso serían, según Rolla y Rivadeneira (2006), el equilibrio entre las actividades dirigidas por la profesora y las de libre elección de los niños; el uso de preguntas y cuestionamientos dirigidos a los niños; un énfasis en el desarrollo del lenguaje y las habilidades cognitivas; enseñanza de elementos de la cultura escolar y un respeto por los alumnos, ofreciéndoles la posibilidad de tomar ciertas decisiones.

Una parte de la calidad de la educación preescolar está determinada por la cantidad de estimulación a la que están expuestos los niños. Meijnen, Lagerweij y de Jong (2003) establecieron que, entre los elementos importantes para recibir una educación de calidad, se encuentran las oportunidades de aprendizaje ofrecidas y el tiempo destinado a las tareas de aprendizaje. A su vez, Ackerman y Barnett (2006) demostraron que la longitud de la jornada tenía un efecto en los aprendizajes, resultando beneficiados quienes asistían a establecimientos con jornadas educativas más prolongadas. Los cambios se presentaron en las competencias de lenguaje oral y escrito y en matemáticas. Es interesante destacar que Ackerman y Barnett (2006) incluyeron escuelas de igual tipo de dependencia, del mismo distrito, que presentaban profesores con similares calificaciones, así como, también, con una cantidad equivalente de alumnos por sala, lo cual refuerza el hallazgo que mayor tiempo instruccional repercute positivamente en el aprendizaje.

De lo anterior se desprende que, si bien la calidad de la enseñanza es un aspecto crítico para el aprendizaje, lo es también el tiempo que se emplea en distintos tipos de actividades. ¿En qué debe invertirse ese tiempo para que se observen los efectos positivos antes citados? De todos los aspectos mencionados anteriormente, el énfasis en la estimulación de las habilidades de lenguaje oral y escrito es uno de los que ha recibido mayor apoyo en la literatura, tanto a nivel teórico como empírico (NICHD, 2000; Ramey \& Ramey, 2006; Rolla \& Rivadeneira, 2006). Uno de los principales aportes que puede hacer la educación preescolar a los niños de sectores desaventajados es proporcionarles oportunidades de desarrollo lingüístico y cognitivo que los dejen mejor preparados para enfrentar los desafíos de la educación primaria. Igualmente, la evidencia procedente de los estudios del desarrollo del lenguaje en infantes y preescolares muestra que existe una relación positiva entre la cantidad de estímulo lingüístico (medido en unidades de tiempo o en número de palabras) y la adquisición de diversos aspectos del lenguaje (adquisición de palabras o construcciones específicas) (Naigles \& Hoff-Ginsberg, 1998; Rowe, 2008; Tomasello, 2006; Valian \& Casey, 2003). Así, la proporción de la jornada preescolar que se dedica a actividades que permiten esta interacción lingüística es relevante para conocer las oportunidades que tienen los niños de estar expuestos al tipo de estimulación requerida para desarrollar su lenguaje. Las actividades en las que el adulto no tiene un rol de interacción verbal con los niños, como el juego libre no supervisado, no ofrecen esta oportunidad a menos que las profesoras o cuidadoras aprovechen estas oportunidades para interactuar con los niños.

Connor et al. (2006) estudiaron las actividades específicas realizadas en las aulas preescolares y su relación con la adquisición de ciertas habilidades de lenguaje, como reconocimiento de las letras y aumento del vocabulario. Sus resultados mostraron que existía una gran variabilidad entre las salas de clases participantes en el estudio, en relación a la cantidad y tipo de oportunidades de aprendizaje. Una mayor cantidad de tiempo instruccional de alta calidad en un preescolar se relaciona con mejores habilidades de lenguaje y alfabetización emergente. En este estudio esta relación fue mayor en los alumnos con un nivel de entrada más bajo, es decir, se beneficiaron mayormente del tiempo instruccional quienes presentaban menores habilidades al inicio. Resultados similares se obtuvieron en los análisis realizados con los datos recogidos por el Early Childhood Longitudinal Study, un estudio masivo de más de 8.000 niños anidados en más de 1.900 salas de kindergarten en Estados Unidos. Este estudio mostró, entre otras cosas, que los niños que asistían a clases donde se le dedicaba más tiempo a la instrucción en 
lenguaje oral y escrito mostraban mayores ganancias durante el año escolar en medidas de alfabetización emergente, aun luego de controlar otros efectos como el tamaño de la clase, la edad del niño, sus habilidades de entrada y su nivel socioeconómico (NSE) (Walston \& West, 2004).

Entre las actividades más importantes de lenguaje que se realizan en el preescolar se cuentan todas aquellas que desarrollan el vocabulario de los niños, especialmente la lectura de cuentos, la enseñanza directa de palabras nuevas y el proveer múltiples oportunidades para que los niños profundicen su comprensión de los significados de las palabras nuevas (Beck, McKeown \& Kucan, 2002) y aquellas que permiten el acercamiento de los niños a elementos del lenguaje escrito -conciencia fonológica, estructura narrativa, usos del lenguaje escrito, alfabeto (McGee \& Richgels, 2003).

Todo lo anterior es consistente con la investigación realizada en otros niveles del sistema escolar, especialmente la enseñanza primaria, en la que también el uso eficiente del tiempo y el énfasis en las habilidades de lenguaje son predictores de la efectividad de una escuela (Bellei, Muñoz, Pérez \& Raczynski, 2003; Martinic, 1998). Estudios realizados en Chile y en el extranjero han mostrado que las escuelas que logran buenos resultados en sectores de pobreza son aquellas donde existe un alto foco en los aprendizajes y en el uso adecuado del tiempo en función de ellos. El tiempo se aprovecharía en estos establecimientos con gran intensidad y de la forma más eficiente posible, estando los alumnos involucrados gran parte del tiempo en actividades de aprendizaje (Bellei et al., 2003; Edmonds, 1979; Maughan, Mortimore, Ouston \& Rutter, 1979).

Sin embargo, pese a la evidencia que existe sobre la importancia de la gestión eficiente del tiempo en la sala de clases, es sabido que esta es un área deficiente en muchos establecimientos educacionales en nuestro país y en otros países latinoamericanos, a nivel primario y secundario. En un estudio sobre el uso del tiempo instruccional en Latinoamérica, Martinic (1998) concluye que existe una gran diferencia entre el tiempo que se asigna oficialmente a la instrucción y el que efectivamente se dedica a ella. Por ejemplo, un estudio en México mostró que un 27\% del tiempo se dedicaba a actividades de enseñanza, mientras que un 33\% se utilizaba en actividades de organización (Ezpeleta \& Weiss, 1994, citado en Martinic, 1998). Parra y Tedesco (1981, citado en Martinic, 1998) observaron en una investigación en Colombia que más del $50 \%$ del tiempo escolar se utilizaba en actividades fuera del aula y en la realización de otras actividades, como la preparación de festivales y actos cívicos. En un estudio realizado en Chile por Himmel (1984, citado en Martinic, 1998) se encontró que las clases tardan en comenzar en promedio nueve minutos y en la mayoría de los casos finalizan por una interrupción.

Los estudios anteriores presentan un cuadro desalentador de la gestión del tiempo en la educación primaria en Latinoamérica. ¿Qué sucede con el uso del tiempo en el aula preescolar? En contraste con el creciente interés en la educación preescolar en nuestro país, se cuenta con poca información acerca de la situación actual de esta. Esto constituye un problema, dado el reciente impulso para desarrollar políticas educativas y sociales que apuntan a la educación preescolar como un mecanismo para fomentar la equidad de oportunidades educativas para los niños de sectores desaventajados. La propuesta de aumentar la cobertura de la educación preescolar, por ejemplo, requiere para su adecuada canalización que los diseñadores de políticas cuenten con información confiable acerca de los factores que pueden afectar la implementación de programas preescolares de calidad en nuestro contexto nacional y su impacto en sectores de pobreza. Sin embargo, lo que sucede a nivel preescolar en nuestro país es en gran medida un misterio para los investigadores, padres y diseñadores de políticas públicas. Sabemos poco acerca de lo que ocurre en las salas preescolares en Chile y Latinoamérica pero, basados en lo que ocurre en educación primaria, podemos suponer que en general el tiempo se gestiona de manera poco efectiva. En Costa Rica, Rolla, Arias y Villers (2005) encontraron que el foco de la instrucción en kindergarten estaba en la socialización y el juego inestructurado, mientras que en Chile, Valenzuela (2005) estudió nueve salas de kindergarten de Santiago, indagando en qué actividades invertían las profesoras de kinder el tiempo destinado a enseñar lenguaje y observó que gran parte de este era utilizado en conversaciones, siendo estas últimas las actividades de lenguaje más frecuentes. Este último estudio incluyó establecimientos de diferente NSE y tipo de dependencia. En un estudio chileno recientemente publicado, Eyzaguirre y Fontaine (2008) dan cuenta del tipo de actividades de lenguaje y lectura y el tiempo dedicado a estas en las salas de clases de kindergarten, $1^{\circ}$ y $2^{\circ}$ básico en 13 escuelas con distintos niveles de desempeño (alto, medio y bajo) en evaluaciones nacionales (SIMCE). Específicamente 
en lo que respecta a kindergarten, las autoras indican que la estimulación del lenguaje es débil en todas las escuelas y el contacto con la lectura es escaso. Por ejemplo, en ninguna de las salas existía un rincón de lectura con libros al alcance de los niños. También encontraron que las diferencias en tiempo de lectura (a favor de las escuelas con más alto SIMCE) eran más acentuadas en kinder y $1^{\circ}$ básico.

Dada la importancia de la instrucción y el tiempo que en ella se utiliza, resulta de gran relevancia profundizar en la investigación respecto a la forma en que se gestiona el tiempo en la educación preescolar en Chile. El presente estudio busca contribuir a formarnos una idea más completa de cómo se gestiona el tiempo en el aula preescolar en Chile, particularmente en el nivel de kindergarten, y comparar lo que está sucediendo en las aulas de kindergarten de nuestro país con lo que sugieren los criterios actuales acerca de lo que debe ofrecer una educación preescolar de calidad. Con esta finalidad, se observaron 12 salas de clases pertenecientes a 9 escuelas de la Región Metropolitana, para determinar a qué actividades y en qué cantidad y frecuencia estaban expuestos los niños durante la jornada pedagógica.

\section{Método}

\section{Participantes}

Las salas de clase participantes fueron reclutadas durante el año 2005 en el contexto de un proyecto mayor (Proyecto FONDECYT No 1040757), y consistieron en 12 aulas de kindergarten y sus profesoras respectivas, pertenecientes a 9 establecimientos educacionales urbanos de la Región Metropolitana. Las aulas se seleccionaron buscando la participación de escuelas de distintos NSE y fuentes de financiamiento, así como distintos resultados en la prueba SIMCE. Así, la muestra intencionada estuvo compuesta como sigue:

1. Cuatro salas de clases pertenecientes a tres establecimientos públicos, dos de ellas de NSE bajo y dos de NSE medio-alto. Uno de los establecimientos obtuvo puntajes SIMCE superiores a los de su grupo socioeconómico y los otros dos, inferiores.

2. Cuatro salas de clases pertenecientes a cuatro establecimientos particulares subvencionados, uno de ellos de NSE medio-alto, dos de
NSE medio-bajo y uno de NSE bajo. De estos establecimientos, tres obtuvieron puntajes SIMCE superiores al promedio de su grupo socioeconómico y el otro obtuvo un puntaje inferior.

3. Cuatro aulas pertenecientes a dos establecimientos privados, ambos de NSE alto. Uno de los establecimientos obtuvo un puntaje SIMCE superior al promedio de su grupo y el otro, inferior.

\section{Instrumento}

Las variables instruccionales de kindergarten fueron medidas utilizando una versión traducida y adaptada de la pauta desarrollada por Connor, Morrison y Griffin (2002), la cual fue diseñada para estimar la cantidad de tiempo destinada a las distintas actividades durante la jornada de clases. La pauta se adaptó en base a aplicaciones piloto que mostraron algunas diferencias con la realidad chilena, y además se incluyó mayor detalle en las actividades de lenguaje, por considerarse uno de los focos más importantes de la instrucción.

La pauta adaptada consta de un total de 63 actividades, agrupadas en las siguientes categorías:

1. Actividades anteriores a la lectura (4 actividades)

2. Actividades de lectura (10 actividades).

3. Actividades posteriores a la lectura (3 actividades)

4. Actividades de alfabeto y conciencia fonológica (4 actividades)

5. Actividades de vocabulario (1 actividad)

6. Actividades de convenciones formales (2 actividades)

7. Actividades de escritura (13 actividades)

8. Actividades de lenguaje oral (11 actividades)

9. Otras actividades instruccionales (7 actividades)

10. Actividades no instruccionales (8 actividades).

La pauta está diseñada para ser aplicada en vivo $o$ en video, y requiere que el observador registre el tiempo de inicio y término de todas las actividades observadas que duren al menos un minuto. Posteriormente, el observador debe decidir a qué actividad de la pauta corresponde cada actividad registrada, $y$ calcular los tiempos totales de cada una. 


\section{Variables}

Las actividades descritas en la pauta se reagruparon en nuevas categorías para los análisis estadísticos, con la finalidad de organizar la información de manera más significativa para el propósito de este estudio. Así, las variables examinadas en este estudio quedaron como sigue:

Actividades instruccionales. Definimos como instruccionales todas aquellas actividades que tienen un propósito de enseñanza o estimulación discernible, y que son dirigidas por uno de los adultos en la sala de clase. Estas incluyen actividades de lenguaje, lectura y escritura (lectura de libros $\mathrm{y}$ todas las actividades relacionadas, actividades con letras y/o sonidos, instrucción de vocabulario, convenciones del lenguaje escrito, actividades de escritura emergente y actividades de lenguaje oral, como discusiones, canciones o poesías), y también otras actividades instruccionales relacionadas con la vida cotidiana (clima, días de la semana, noticias), matemáticas (precálculo, gráficos y geometría), manualidades, motricidad fina y gruesa, arte y percepción. Cuando los niños trabajaban en pequeños grupos, se clasificó el tiempo como instruccional si al menos uno de los grupos estaba involucrado en una actividad instruccional.

Actividades no instruccionales. Definimos como no instruccionales aquellas actividades en la cuales no es posible distinguir ninguna intencionalidad de enseñanza o estimulación, y donde los niños están involucrados en actividades no dirigidas por adultos. Solo se clasificó el tiempo como no instruccional cuando esto era aplicable al grupo completo de niños. Se incluyen en esta categoría el control de la conducta de los niños, colación, limpieza y baño, juego libre y recreo, así como otras actividades sin un claro fin instruccional que no se clasifican dentro de las ya detalladas.

Actividades de lenguaje. Dentro de las actividades instruccionales se realizó un acercamiento a las actividades de lenguaje. Estas incluyen las siguientes actividades:

1. Lenguaje oral. Incluye conversaciones, canciones, poesías, señales, actividades fono articulatorias simples y complejas, títeres, dramatizaciones, disertaciones, historias orales y actividades relacionadas con otra lengua.
2. Lectura. Agrupa la introducción a la lectura, convenciones sobre el libro, lectura que realiza la profesora con y sin discusión, escuchar grabaciones, lectura grupal, lectura coral global y grupal, lectura en forma global o grupal, lectura interactiva, lectura silenciosa sostenida. A su vez, incluye discusión sobre el texto leído y trabajos o actividades desarrolladas después de la lectura.

3. Alfabeto y conciencia fonológica. En estas actividades se incluyen el nombre y sonido de las letras, el sonido inicial de palabras y segmentación y síntesis de palabras.

4. Vocabulario. Se incluyen las actividades en que la profesora y los niños discuten sobre el significado de una palabra.

5. Escritura. Agrupa actividades introductorias a la escritura, de apresto motor, la escritura de palabras, frases, cuentos y dictados. También se incluyen actividades como ordenar palabras escritas, copiar frases u oraciones, releer lo escrito, dictar frases o ideas que la profesora va escribiendo y la realización de lluvia de ideas cuando se orienta a la escritura.

6. Convenciones formales. Entre estas actividades se encuentra la instrucción y ejercitación de convenciones formales, tales como direccionalidad, métrica de poemas y signos de puntuación.

\section{Procedimiento}

Todas las observaciones fueron grabadas en video con el fin de obtener información de confiabilidad entre los codificadores.

El contacto original con los colegios se hizo a través de los directores o directoras. Posteriormente, en una entrevista con las profesoras, se les explicó el procedimiento y se les solicitó su consentimiento. A pesar que no se les solicitó firmar un consentimiento, los padres fueron informados en una reunión de apoderados acerca de las filmaciones en sala y se les aseguró que estas solo serían utilizadas por personal del proyecto con fines de investigación. Estas filmaciones fueron hechas al nivel de la clase completa, focalizadas en la profesora y no en algún niño en particular, sin variar el ángulo de la cámara. Por esta razón, suponen una invasión mínima de la privacidad de los niños.

Aunque el diseño original contemplaba tres observaciones en cada sala de clase, esto no fue posible en todas las salas, debido a problemas variados, 
como ausentismo de las profesoras o actividades especiales no programadas. Así, una de las 12 salas fue observada solo una vez, mientras que en otra aula se realizaron solo dos observaciones. En todas las restantes se efectuaron tres observaciones, resultando un total de 33 observaciones. Las tres observaciones se realizaron con un espacio aproximado de un mes entre cada una, entre los meses de Julio y Diciembre. La mayor parte de las observaciones abarcaron una jornada instruccional completa (ya sea una mañana o una tarde), aproximadamente tres horas desde que los niños llegan a la sala hasta que se retiran. Dos salas de clase tenían un horario extendido, por lo que la observación se finalizó a la hora del almuerzo para equiparar el tiempo de observación con las demás salas. En dos ocasiones las profesoras solicitaron que la observación concluyera más temprano, por lo cual hay tres observaciones de una hora de duración cada una.

Todas las observaciones fueron codificadas en vivo por una observadora entrenada y filmadas en video para un posterior análisis de confiabilidad. La confiabilidad inter-codificadoras fue muy alta, alcanzándose una correlación ${ }^{1}$ de al menos 0,90 entre codificador en vivo y en video para todas las categorías observadas (algunas no se dieron nunca), luego de tomar en cuenta algunos lapsos en la filmación.

\section{Resultados}

Los análisis se efectuaron utilizando la observación como unidad de análisis $(n=33)$, y no la sala de clase $(n=12)$, para obtener mayor potencia. La duración promedio de las jornadas observadas fue de 196,79 minutos con una desviación estándar de 56,41 minutos. De las 33 observaciones, 24 tuvieron una duración de tres horas o más (cinco de ellas duraron un poco más de cuatro horas). De las nueve observaciones restantes, seis duraron 150 minutos o más y tres fueron de tan solo una hora. Dada la variabilidad de la duración de las observaciones, los resultados descriptivos se presentan tanto en valores brutos (minutos) como en proporción del tiempo total observado, para facilitar la interpretación.

La Tabla 1 resume el tiempo dedicado a actividades instruccionales y no instruccionales, según el tipo de dependencia del establecimiento.

Tabla 1

Promedio de Tiempo Dedicado a Actividades Instruccionales y No Instruccionales, según Tipo de Dependencia del Establecimiento, en Minutos y en Proporción del Tiempo Observado

\begin{tabular}{|c|c|c|c|c|c|c|c|c|c|}
\hline \multirow{2}{*}{ Tipo de Actividades } & & \multicolumn{2}{|c|}{ Municipal } & \multicolumn{2}{|c|}{$\begin{array}{c}\text { Particular } \\
\text { Subvencionado }\end{array}$} & \multicolumn{2}{|c|}{ Particular Pagado } & \multicolumn{2}{|c|}{ Total } \\
\hline & & Media & $D E$ & Media & $D E$ & Media & $D E$ & Media & $D E$ \\
\hline \multirow{2}{*}{$\begin{array}{l}\text { Actividades } \\
\text { Instruccionales }\end{array}$} & Min & 95,36 & 25,45 & 99,80 & 41,31 & 73,50 & 53,88 & 88,75 & 42,67 \\
\hline & Prop & 0,55 & 0,11 & 0,51 & 0,17 & 0,37 & 0,25 & 0,47 & 0,20 \\
\hline \multirow{2}{*}{$\begin{array}{l}\text { Actividades } N o \\
\text { Instruccionales }\end{array}$} & Min & 83,09 & 31,27 & 101,10 & 41,61 & 136,75 & 65,70 & 108,06 & 53,05 \\
\hline & Prop & 0,45 & 0,11 & 0,49 & 0,17 & 0,63 & 0,25 & 0,53 & 0,20 \\
\hline
\end{tabular}

Nota. Valores expresados en minutos (Min) y en proporción del tiempo total observado (Prop).

$n$ Municipales $=11 ; n$ Particulares Subvencionados $=10 ; n$ Particulares Pagados $=12$.

Como se observa en la Tabla 1, el tiempo dedicado a actividades instruccionales es escaso, siendo

Debido a que la variable de interés es continua (minutos), para estimar la confiabilidad entre las codificadoras se utilizó la correlación lineal de Pearson entre los minutos estimados por las dos codificadoras para cada categoría de actividades. el promedio de todos los establecimientos de casi 89 minutos ( $47 \%$ del tiempo total observado). Es decir, en una mañana típica de tres horas, menos de una hora y media es dedicada a actividades que pueden ser llamadas instruccionales (con un criterio amplio, ya que incluimos entre ellas incluso actividades cuyo fin instruccional puede ser difuso, tales como juegos dirigidos, manualidades y conversaciones). El resto, 
un promedio de 108 minutos, es dedicado a actividades no instruccionales, incluyendo recreo, colación (merienda) y el control de la conducta de los niños.

La Tabla 1 sugiere que podría haber diferencias en la cantidad de minutos dedicados a actividades instruccionales y no instruccionales entre los establecimientos de distinto tipo de dependencia: superior en los colegios particulares pagados, seguidos de los colegios subvencionados, siendo los municipales los establecimientos que dedican menor tiempo a actividades no relacionadas con la instrucción. Con el fin de determinar si estas diferencias son significativas, se realizó un análisis de varianza. Este reveló que no existen diferencias significativas por tipo de dependencia en el promedio de minutos dedicados a actividades instruccionales, $F(2,30)=1,25, p=0,3$. Sin embargo, sí existe una diferencia en relación
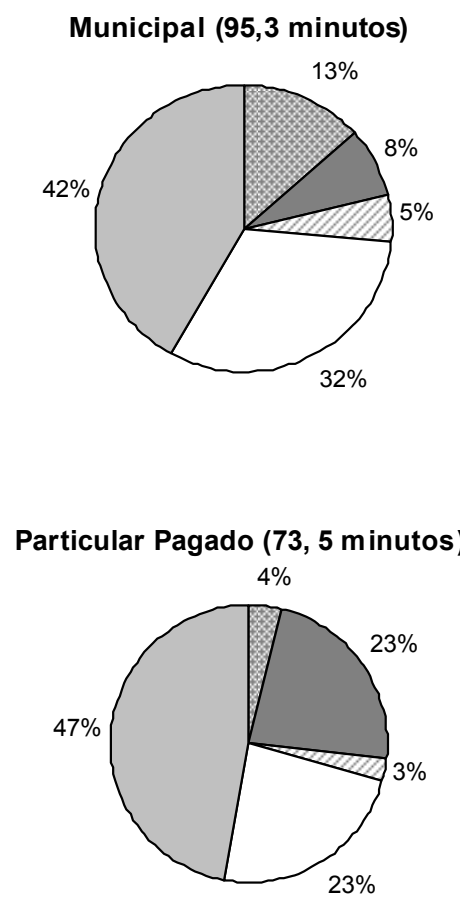

$$
\text { @ Matemáticas } \square \text { Otras } \square \text { Vida cotidiana } \square \text { Manualidades } \square \text { Lenguaje, Lectura y Escritura }
$$

Figura 1. Distribución del tiempo instruccional según tipo de dependencia del establecimiento y en la muestra total. a las actividades no instruccionales, $F(2,30)=$ $3,55, p=0,04$. Las pruebas post-hoc muestran que en las observaciones realizadas en colegios particulares se registró significativamente un mayor tiempo dedicado a actividades no instruccionales que en las observaciones realizadas en los colegios municipales (Bonferroni, $p=0,04$ ). Entre los colegios municipales y particulares subvencionados no existen diferencias significativas en esta variable ni tampoco entre estos últimos y los colegios particulares pagados.

Luego de determinar cuánto tiempo de la jornada preescolar se dedica a actividades instruccionales y no instruccionales, realizamos un acercamiento a la forma en que se distribuye el tiempo que sí se dedica a instrucción. En la Figura 1 se desagrega el tiempo instruccional en distintos tipos de actividades.

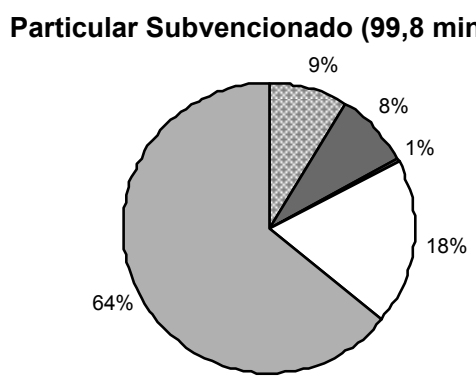

Muestra Total $(88,75$ minutos)

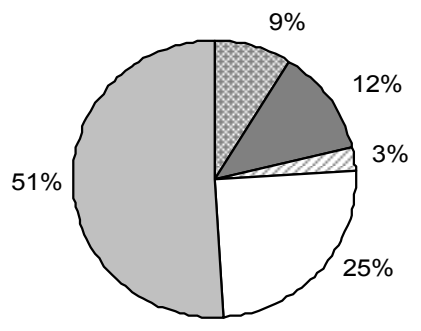


Los resultados muestran que entre las actividades instruccionales, la mayor parte del tiempo se dedica a actividades de lenguaje en los establecimientos de todo tipo de dependencia. Los colegios municipales y particulares pagados invierten un monto de tiempo muy similar en este tipo de actividades, diferenciándose de los colegios particulares subvencionados de la muestra, quienes superan aproximadamente en $20 \%$ el tiempo que los otros establecimientos dedican al lenguaje.

Las manualidades ocupan parte importante de la jornada en las aulas de kindergarten. En los colegios de todo tipo de dependencia estas actividades ocupan el segundo lugar en la cantidad de tiempo invertido. El tiempo dedicado a actividades matemáticas es bajo en los tres tipos de dependencia. Los colegios municipales fueron quienes invirtieron mayor cantidad de tiempo en el desarrollo de estas actividades, seguidos por los colegios particulares subvencionados y los particulares pagados, quienes dedicaron menos de un $10 \%$ del tiempo instruccional a estas actividades. Los colegios particulares pagados dedicaron un porcentaje importante del tiempo instruccional a

Municipal (39,9 minutos)

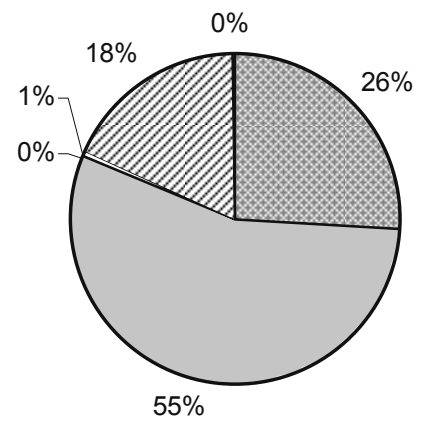

Particular Pagado (34,7 minutos)

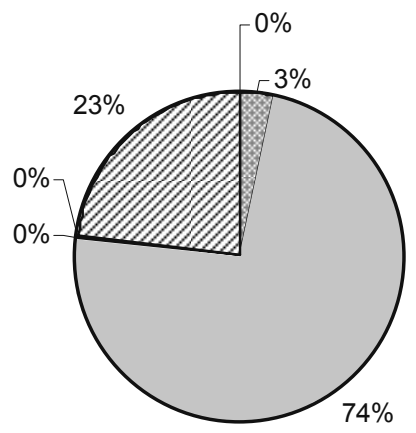

otras actividades instruccionales, dentro de las cuales se incluyen, por ejemplo, computación, trabajo en afectividad y educación física. El tiempo utilizado supera al invertido en matemáticas y en actividades relacionadas con la vida cotidiana.

Con el fin de determinar si existía alguna diferencia significativa en la distribución del tiempo instruccional en los distintos colegios, se realizó un análisis de varianza. Los resultados muestran que no existen diferencias significativas en la cantidad de tiempo que se dedica a las distintas actividades instruccionales en los colegios según su tipo de dependencia, exceptuando una diferencia significativa entre el tiempo destinado a la vida cotidiana entre los colegios municipales y los particulares subvencionados, a favor de los primeros, $F(2,30)=3,75, p=0,04$. Las pruebas post hoc confirman la significación de esta diferencia (Bonferroni, $p=0,041$ ).

A continuación nos enfocamos con mayor detalle en el tipo de actividades de lenguaje, lectura y escritura que se realizan durante los 45 minutos promedio que se dedican a esta área. Esto se muestra en la Figura 2.

\section{Particular Subvencionado (64 minutos)}

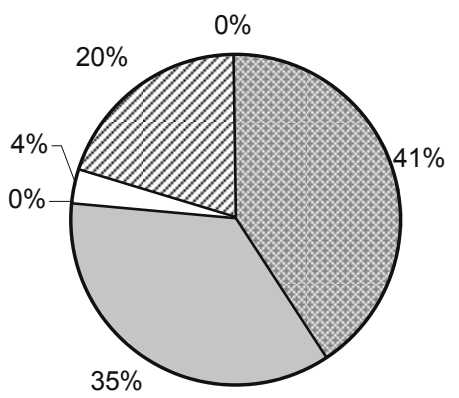

Muestra Total (45,3 minutos)

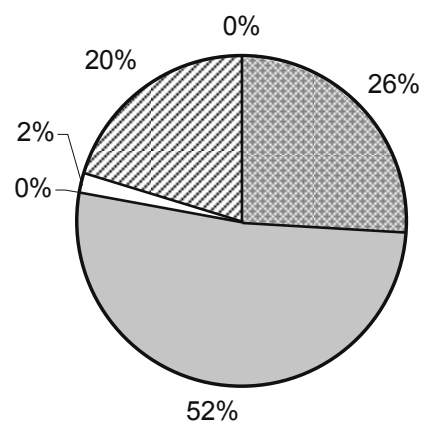

曰Lectura $\quad$ Vocabulario $\square$ Alfabeto y Conciencia Fonológica $\quad$ Escritura $\square$ C̣onvenciones

Figura 2. Distribución del tiempo dedicado a Lenguaje según dependencia del establecimiento y en la muestra total. 
Como se observa en la Figura 2, las actividades de lenguaje más frecuentes en todos los establecimientos son las de lenguaje oral (conversaciones, canciones, poesías). El tiempo dedicado a lectura de libros y actividades relacionadas es poco. En promedio, sin tomar en cuenta el tipo de establecimiento, las profesoras de kindergarten de esta muestra invierten apenas 11,84 minutos de la jornada en actividades relacionadas con libros (incluyendo no solo leer un cuento sino también conversar sobre él, hacer inferencias, hablar sobre su vocabulario, etc.). Al desglosar esta categoría, pudimos constatar que el tiempo dedicado a la lectura propiamente tal, excluyendo manualidades y juegos asociados al libro leído, es de tan solo 5 minutos en promedio. Otra actividad que está notoriamente ausente es la instrucción en vocabulario, la cual no ocurre ni en forma aislada ni en el contexto de la lectura de cuentos. En las 33 observaciones que se analizan en este estudio, no se observó en ninguna ocasión que las profesoras discutieran con los niños el significado de una palabra. Asimismo, en promedio solo el 2\%

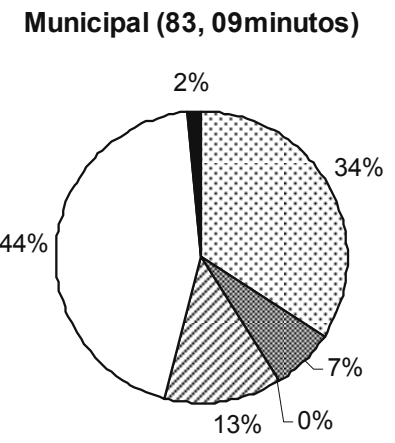

Particular Pagado (136,7 minutos)

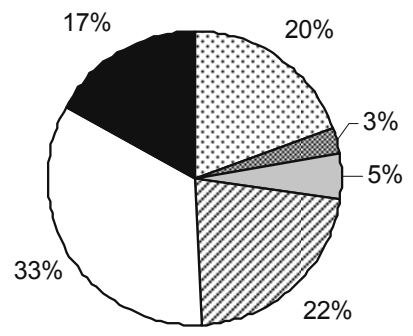

del tiempo dedicado a lenguaje se utiliza en aprender el sonido o nombre de las letras o en analizar los sonidos del lenguaje, lo que equivale a menos de un minuto por jornada.

Un análisis de varianza mostró que existen diferencias en la cantidad de tiempo destinado a la lectura y sus actividades asociadas, $F(2,30)=$ $5,33, p=0,01$. La prueba post hoc (Bonferroni, $p=$ $0,009)$ confirmó que entre los colegios particulares pagados y los particulares subvencionados existe una diferencia significativa en cuanto al tiempo destinado a las actividades relacionadas con libros, a favor de los segundos.

Finalmente, debido a la gran cantidad de tiempo que se invertía en actividades no instruccionales en esta muestra, decidimos realizar también un acercamiento a estas actividades, para determinar en qué específicamente se invierte la enorme cantidad de tiempo que no se dedica a enseñar o estimular a los niños. La Figura 3 representa la distribución del tiempo no instruccional en sus actividades componentes.

\section{Particular Subvencionado (101, 1 minutos)}

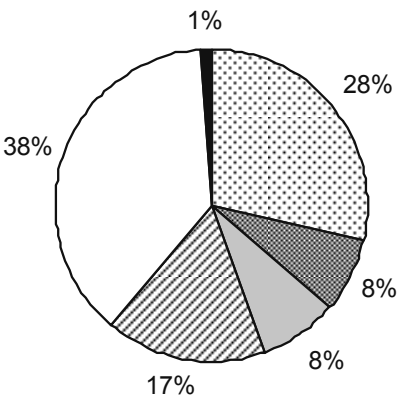

Total (108,07 minutos)

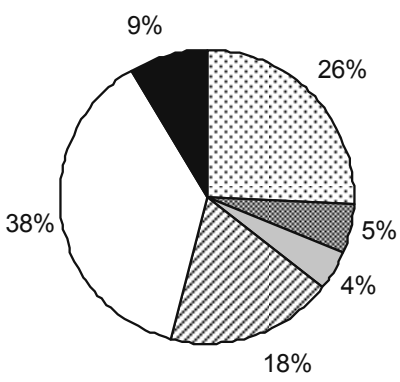
๑Manejo
圆 Hábitos
$\square$ Juegos
$\square$ Colación
$\square$ Recreo
Otros

Figura 3. Distribución del tiempo no instruccional por tipo de dependencia y en la muestra total. 
Como puede verse, la mayor parte del tiempo no instruccional es utilizado en recreo, siendo cercana la cantidad de tiempo destinada a controlar la conducta de los alumnos. La colación o merienda es otra actividad que ocupa una parte importante del tiempo no instruccional de la jornada. La categoría otros, que explica una parte no despreciable del tiempo en los colegios particulares pagados, agrupa actividades como la preparación de actos para ocasiones especiales, despedidas, preparación de los niños para irse a su casa, reuniones y fiestas religiosas. El análisis de varianza no mostró diferencias significativas por tipo de dependencia en estas variables.

\section{Discusión}

Los resultados de este estudio replican para la educación preescolar los resultados encontrados por otros autores en la enseñanza primaria: la gestión del tiempo en estas aulas preescolares no es buena, independientemente del tipo de dependencia del establecimiento. Una gran porción de la jornada escolar se pierde, dedicada a actividades no instruccionales. En este estudio ampliamos lo más posible la definición de lo que se considera instruccional, por tratarse de un estudio en enseñanza preescolar y conociendo las diversas visiones que existen sobre los objetivos y funciones de la educación preescolar. Tomando en cuenta que uno de los principales determinantes del impacto de los programas preescolares es la cantidad de lenguaje dirigida a los niños (NICHD, 2000), codificamos como actividades instruccionales todas las que involucraran lenguaje. Incluimos así actividades tan inestructuradas como el juego de libre elección en rincones (donde las profesoras interactuaban ocasionalmente con los niños en forma individual a propósito de sus juegos), las conversaciones grupales sobre eventos cotidianos y las canciones. Aun así, la gran mayoría del tiempo fue dedicado a juego inestructurado, es decir, no supervisado por un adulto. Este tiempo, por lo tanto, no ofrecía la posibilidad de interactuar lingüísticamente con los niños.

El detalle de lo que hacen las profesoras durante los minutos que sí están dedicados a enseñar tampoco es alentador. Las actividades que sabemos son esenciales para que la educación preescolar tenga un impacto positivo sobre el desarrollo cognitivo y el futuro rendimiento escolar están notoriamente ausentes de la jornada de kindergarten en la muestra. Por una parte, no observamos instrucción alguna en vocabulario, ni explícita ni implícita. Es decir, en las 33 ocasiones en que visitamos las salas, durante tres o cuatro horas en la mayoría de los casos nunca observamos a una profesora discutir con los niños el significado de alguna palabra. El tiempo invertido en estudiar el alfabeto (aprender los nombres o sonidos de las letras) y en analizar los sonidos del lenguaje hablado (conciencia fonológica) también fue muy escaso, aun cuando estos conocimientos y habilidades son esenciales para el posterior aprendizaje de la lectura y la escritura. En forma igualmente preocupante, pudimos constatar que en esta muestra el tiempo dedicado a interactuar con libros en general ocupó una porción insignificante de la jornada. Al sumar los tiempos dedicados a escuchar la lectura de un libro, discutir sobre un texto leído o por leer, e incluso hacer manualidades relacionadas con un texto que se leyó, el tiempo apenas supera los 11 minutos. Estos hallazgos coinciden con lo reportado por Eyzaguirre y Fontaine (2008), quienes, además de constatar el escaso tiempo dedicado a actividades relacionadas con la lectura, reportan que al entrevistar a las educadoras estas revelan que, en general, la expansión del vocabulario y la estimulación del lenguaje no figuran entre sus prioridades.

Estos resultados son especialmente desalentadores si consideramos que el estudio se realizó en el nivel de kindergarten, el cual debiera constituir una transición entre la enseñanza preescolar y la enseñanza escolar. ¿A qué se debe esta situación? Una razón posible para este uso laxo del tiempo en esta muestra es lo que se espera en general de los centros preescolares en nuestra región y, en consecuencia, cómo se define lo que califica como un buen uso del tiempo. Sabemos que en algunos círculos se visualiza la enseñanza directa en el preescolar como excesivamente "escolarizadora", como una actividad que podría limitar el desarrollo integral del niño o la expresión plena de su potencial (Rolla et al., 2005). Esta creencia no tiene fundamento alguno de carácter científico. Por el contrario, las teorías modernas del desarrollo psicológico, así como los hallazgos recientes sobre el desarrollo y el aprendizaje en los primeros años, nos muestran una imagen de un niño preescolar ávido por aprender, que busca activamente situaciones en las que pueda poner en juego sus amplias capacidades de construcción de conocimiento (Gopnik, Meltzoff \& Kuhl, 2000). La estructuración de la situación de aprendizaje, la dirección por parte de un adulto y la instrucción explícita, en lugar de truncar esta tendencia natural, la potencian. Enseñanza explícita e instrucción no son sinónimos de enseñanza ex- 
positiva y aprendizaje receptivo, tal como el juego inestructurado y la colación no son lo mismo que un ambiente propicio para el desarrollo integral del niño y su expresión plena.

Estos resultados son consistentes con el estado de la educación chilena. El tiempo en la sala de clase se invierte en actividades que no tienen ningún objetivo instruccional para los niños, y el resultado es el esperable. Más aún, el hecho que la gestión del tiempo en los diferentes establecimientos sea similar, independientemente de su dependencia, sugiere que las diferencias en los resultados educativos de niños de colegio municipales y subvencionados no se deben a algo que los colegios hacen distinto. Los resultados de este estudio muestran que, al menos en esta pequeña muestra y al nivel de kindergarten, los colegios municipales no son peores que los particulares en cuanto a la cantidad de instrucción que ofrecen, por lo cual cualquier diferencia en el rendimiento de fin de año que se observe a favor de los colegios particulares, no podría eventualmente explicarse por diferencias en la gestión de las aulas entre los tres tipos de establecimientos. Esto es consistente con lo mostrado por datos de evaluaciones a gran escala como SIMCE y PISA, en los que, al controlar por características de entrada de los niños, especialmente su NSE, los tres tipos de escuelas obtienen resultados similares, sugiriendo que las diferencias brutas en los resultados de los tres tipos de dependencia se deben más a las diferencias de entrada de los niños (Manzi, Strasser, San Martín \& Contreras, 2008).

Las consecuencias de la mala gestión del tiempo observada en el nivel de kindergarten no pueden ser positivas. Así como sabemos que un buen uso del tiempo del aula primaria es un factor clave para la efectividad de un profesor, también hay datos que indican que el tiempo invertido en actividades instruccionales y la cantidad de lenguaje dirigida a los niños en kindergarten es uno de los determinantes de su impacto positivo sobre la preparación del niño para enfrentar la instrucción en primero básico (Connor et al., 2006; Strasser \& Lissi, en prensa; Walston $\&$ West, 2004). Hace falta invertir una cantidad importante de tiempo en estimular el lenguaje oral de los niños, en especial su adquisición de nuevo vocabulario y sus habilidades narrativas, y también en familiarizarlos con los aspectos centrales del lenguaje escrito: conocimiento del alfabeto, conciencia fonológica, estructura de las historias, convenciones de lo escrito y lenguaje descontextualizado. Estas son habilidades que formarán una base sólida para la instrucción lectora en primero básico y que todos los niños pueden desarrollar paulatinamente desde muy temprano, en formas apropiadas según cada etapa del desarrollo. En una sociedad letrada, los niños pueden -y deben- acercarse desde el nacimiento al mundo del lenguaje escrito, tanto en su hogar como en las instituciones educativas a las que asisten. Privarlos de estas oportunidades representa un enorme daño para su desarrollo futuro.

Si la educación preescolar chilena aspira a responder a las altas expectativas depositadas hoy en día en ella por las autoridades educativas y por la población en general, es urgente que esta ofrezca oportunidades reales de aprendizaje y estimulación para los niños, y que se convierta, por ende, en mucho más que un ambiente cálido y protegido donde nuestros niños pasan el tiempo en recreo, colación y un poco de instrucción.

Como una limitación de los resultados reportados, es importante tomar en cuenta las características de la muestra. Tanto el reducido número de escuelas como su homogeneidad (todas pertenecientes a la Región Metropolitana y, por ende, todas urbanas) no permiten generalizar estos resultados al resto de las salas de kindergarten del país. Sin embargo, la selección que se hizo de las 12 salas para incorporar diferentes dependencias, NSE y puntajes SIMCE, así como la consistencia de nuestros resultados con los de otros estudios latinoamericanos (Eyzaguirre \& Fontaine, 2008; Rolla et al, 2005; Valenzuela, 2005), sugieren que lo observado en esta muestra podría reflejar la situación de muchas otras salas chilenas de kindergarten.

Que todas las salas de kindergarten de la muestra pertenezcan a establecimientos educacionales de educación primaria, introduce otro sesgo adicional. Estas salas podrían ser diferentes a las salas de kinder existentes en centros dedicados exclusivamente a la educación preescolar (jardines infantiles), sesgando los resultados en varios sentidos posibles. Es posible, por ejemplo, que las profesoras de kinder de escuelas primarias tiendan a diferenciarse del resto de la escuela, conduciendo sus aulas de una manera que ellas conciben como menos escolarizada, lo cual podría sesgar nuestros resultados hacia un menor tiempo instruccional promedio que el existente en la población general. Por otro lado, también podría ser que las profesoras preescolares de establecimientos de educación primaria, comparadas con las de jardines infantiles, hagan precisamente lo contrario, es decir, que aproximen su instrucción a lo que se hace en primero básico, en cuyo caso 
nuestros resultados en realidad sobreestimarían la cantidad de instrucción que se brinda en la población general de aulas de kindergarten. Dados los datos disponibles en este estudio, es imposible saber si la muestra de profesoras de kinder que trabajan en escuelas primarias es representativa de la población de profesoras de kinder, o si existe alguna diferencia con las profesoras que se desempeñan en jardines infantiles.

\section{Referencias}

Ackerman, D. \& Barnett, S. (2006). Increasing the effectiveness of preschool programs. New Brunswick, NJ: National Institute for Early Education Research. Extraído el 15 Enero, 2007, de http://nieer.org/resources/research/IncreasingEffectiveness. pdf

Beck, I. L., McKeown, M. G. \& Kucan, L. (2002). Bringing words to life: Robust vocabulary instruction. New York: Guilford Press.

Bellei, C., Muñoz, G., Pérez, L. M. \& Raczynski, D. (2003). Escuelas efectivas en sectores de pobreza ¿Quién dijo que no se puede? En R. Hevia (Ed.), La educación en Chile, hoy (pp. 55-78). Santiago, Chile: Ediciones Universidad Diego Portales.

Connor, C. M., Morrison, F. J. \& Griffin, E. (2002). Measuring classroom instruction. Manuscrito no publicado, University of Michigan Pathways to Literacy Project, Ann Arbor, MI, Estados Unidos.

Connor, C. M., Morrison, F. J. \& Slominski, L. (2006). Preschool instruction and children's emergent literacy growth. Journal of Educational Psychology, 98, 665-689.

Edmonds, R. R. (1979). Effective schools for the urban poor. Educational Leadership, 37(1), 20-24.

Eyzaguirre, B. \& Fontaine, L. (2008). Las escuelas que tenemos. Santiago, Chile: Centro de Estudios Públicos.

Gopnik, A., Meltzoff, A. N., \& Kuhl, P. K. (2000). The scientist in the crib: What early learning tells us about the mind. New York: HarperCollins.

Manzi, J., Strasser, K., San Martín, E. \& Contreras, D. (2008). Quality of education in Chile. Santiago, Chile: Centro de Medición de la Pontificia Universidad Católica de Chile (Mide UC).

Martinic, S. (1998). Tiempo y aprendizaje (World Bank LCSHD Paper Series No 26). Washington, DC: The World Bank Latin America and the Caribbean Regional Office.

Maughan, B., Mortimore, P., Ouston, J. \& Rutter, M. (1979).
Fifteen thousand hours. London: Open Books.

McGee, L. M. \& Richgels, D. J. (2003). Designing early literacy programs: Strategies for at-risk preschool and kindergarten children. New York: Guilford Press.

Meijnen, G. W., Lagerweij, N. W. \& de Jong, P. F. (2003). Instruction characteristics and cognitive achievement of young children in elementary schools. School Effectiveness and School Improvement, 14, 159-187.

Naigles, L. R. \& Hoff-Ginsberg, E. (1998). Why are some verbs learned before other verbs? Effects of input frequency and structure on children's early verb use. Journal of Child Language, 25, 95-120.

National Institute of Child Health and Human Development (2000). The relation of child care to cognitive and language development. Child Development, 71, 960-980.

Ramey, S. L. \& Ramey, C. T. (2006). Early educational interventions: Principles of effective and sustained benefits from targeted early education programs. En D. K. Dickinson \& S. B. Neuman (Eds.), Handbook of early literacy research (Vol. 2, pp. 445-459). New York: Guilford Press.

Rolla, A., Arias, M. \& Villers, R. (2005). Quality early childhood education in Costa Rica? Policy, practice, outcomes and challenges. Early Years, 25, 113-127.

Rolla, A. \& Rivadeneira, M. (2006). ¿Por qué es tan importante y cómo es una educación preescolar de calidad? Santiago, Chile: Expansiva. Extraído el 16 Junio, 2008, de http://www. expansiva.cl/media/en_foco/documentos/19062006104123. pdf

Rowe, M. (2008). Child-directed speech: Relation to socioeconomic status, knowledge of child development and child vocabulary skill. Journal of Child Language, 35, 185-205.

Strasser, K. \& Lissi, M. R. (en prensa). Home and instruction effects on emergent literacy in a sample of Chilean kindergarten children. Scientific Studies of Reading.

Tomasello, M. (2006). Acquiring linguistic constructions. En D. Kuhn \& R. Siegler (Eds.), Handbook of child psychology (pp. 255-298). New York: Wiley.

Valenzuela, F. (2005). Instrucción temprana y alfabetización inicial: estudio en niños de una comuna de Santiago. Tesis no publicada para obtener el grado de Magíster en Psicología con mención en Educación, Pontificia Universidad Católica de Chile, Santiago, Chile.

Valian, V. \& Casey, L. (2003). Young children's acquisition of wh-questions: The role of structured input. Journal of Child Language, 30, 117-143.

Walston, J. T \& West, J. (2004). Full-day and half-day kindergarten in the United States. Findings from the Early Childhood Longitudinal Study, Kindergarten Class of 1998-99. Washington, DC: National Center for Education Statistics, Department of Education.

Fecha de recepción: Octubre de 2008.

Fecha de aceptación: Enero de 2009. 